\title{
3D Hole Inspection Using Lens with High Field Curvature
}

\author{
Petr Zavyalov \\ Technological Design Institute of Scientific Instrument Engineering, Siberian Branch of the Russian Academy of Sciences \\ 41, Russkaya str., Novosibirsk, 630058, Russia \\ e-mail: zavyalov@tdisie.nsc.ru
}

\begin{abstract}
One of the actual 3D measurement problems is the optical inspection of various holes. In this respect, the task of plane image formation of holes as extended 3D objects using optical methods turns out to be of primary importance. We have developed specialized lenses that perform such transformations due to specially increased aberrations (field curvature, astigmatism) for the formation of extended objects plane images. The calculations of the lens parameters are presented. The detail analysis of the imaging properties was carried out. The presented hole inspection lens has been designed, constructed and used for inspection of the fuel assembly spacer grids.
\end{abstract}

Keywords: Hole inspection lens, 3D imaging, field curvature, distortion, fuel assembly, spacer grid.

\section{INTRODUCTION}

3 INSPECTION of various holes is the actual measurement problem. Among the noncontact methods for inspection of such objects, the most promising are the optical ones due to high informativeness. They allow inspecting both the geometric parameters of holes and the quality (i.e. appearance) of the surfaces. In this respect, the task of plane images formation of holes as extended 3D objects using optical methods turns out to be of primary importance. Various methods of mechanical scanning and unfolding are traditionally used for this purpose, but they have significant disadvantages, first of all low speed and elaborate design [1-5].

Earlier we have proposed the use of specialized lenses with specially increased aberrations (field curvature, astigmatism) for formation of extended holes plane images [6]. Using such lenses seems a rather promising solution that allows one to simplify the optical schemes for machine vision systems substantially and to expand their performance capabilities.

It should be noted that recently the hole inspection lenses appear on the optical systems market [7,8]. As an example we can note the PCHI-lens from the Opto Engineering Company for inspecting the holes with a diameter from 10 to $120 \mathrm{~mm}$ with a ratio of their length to diameter about unity.

However, the information about the operation principle of such lenses, their optical characteristics and methods of calculation is absent in the scientific literature.

The calculations of the lens parameters are presented in the given article. This hole inspection lens (HIL) has been designed, constructed and used for inspection [9] of the fuel assembly spacer grids. The method for calculating and designing such lens with the help of ZEMAX [10] software package is presented and the imaging properties of this lens are estimated.

\section{A THIN LENS APPROACH FOR A HOLE IMAGE FORMATION}

Firstly, let us analyze the imaging properties of an ideally thin lens for a cylindrical hole formation (Fig.1.). This approach will help us to demonstrate the difference between HIL and conventional lenses. The lens with focal length $f^{\prime}$ is located outside the hole with diameter $D_{h}$ and length $L_{h}$, while an object is a longitudinal extra-axial segment $A B$.

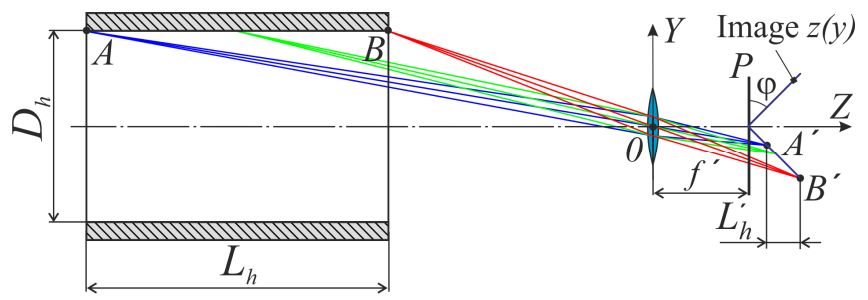

Fig.1. The image formation of the cylindrical hole by a thin lens $\left(f^{\prime}=5 \mathrm{~mm}, D_{h}=10 \mathrm{~mm} \rightarrow \varphi=45^{\circ}\right)$.

As one can see from Fig.1., the longitudinal segment $A B$ is projected by a thin lens into an inclined segment $A^{\prime} B^{\prime}$. Essentially, the image of a cylindrical surface will be conical. According to the thin lens formula, the segment image configuration is described in this case by the expression:

$$
z(y)=\frac{2 f^{\prime}}{D_{h}}|y|+f^{\prime}=\operatorname{tg}(\varphi) \cdot|y|+f^{\prime}
$$

It can be seen that the conical image curvature depends on the ratio $f^{\prime} / D_{h}$. And the cone angle $(\pi-2 \varphi)$ increases with the increase of the ratio $f^{\prime} / D_{h}$. It means that the thin lenses (really standard lenses) may be used for formation of hole images in detector plane $P$ under condition $f^{\prime}<<D_{h}$, i.e. when large holes, for instance, with a diameter about $100 \mathrm{~mm}$ and more, are inspected. In this case the conical image of a hole tends to plane $P(\varphi \rightarrow 0)$. This method for holes or closed cavity image formation is used in endoscopes of various purposes where the focal length of objectives is about some millimeters.

However, using this approach is not feasible for products with smaller diameter holes (less than $100 \mathrm{~mm}$ ) due to the fact that there are considerable difficulties in the production of ultra-short-focus lenses (less than $5 \mathrm{~mm}$ ) and there are no 
matrix photodetectors of suitable sizes (the size of most sensors is about $5-15 \mathrm{~mm}$ ).

Another approach for forming the hole images is based on the use of conic mirror [1-5], that is located inside the inspected hole. In this case to obtain the image of the entire object the mechanical scanning is usually used, which is unacceptable in case of high inspection productivity.

\section{THE FORMATION OF HOLE PLANE IMAGE BY THE SPECIALIZED INSPECTION LENS}

It is evident that using a standard lens that projects a plane image on a plain surface does not allow forming the high quality image of an extended hole with a small diameter. For this purpose it is necessary to design a specific lens, that could be described as a lens for hole or inner surfaces inspection.

The essence of the transformation performed by this lens is illustrated by Fig.2. The lens projects the cylindrical surface of the hole with the diameter $D_{h}$ into the plane of a photodetector sensor. That means the lens has to project the longitudinal segment $A B$ (with coordinates $z_{\min }$ and $z_{\max }$ ) into a transversal segment $A^{\prime} B^{\prime}$ (with coordinates $R_{\min }$ and $R_{\max }$ ).

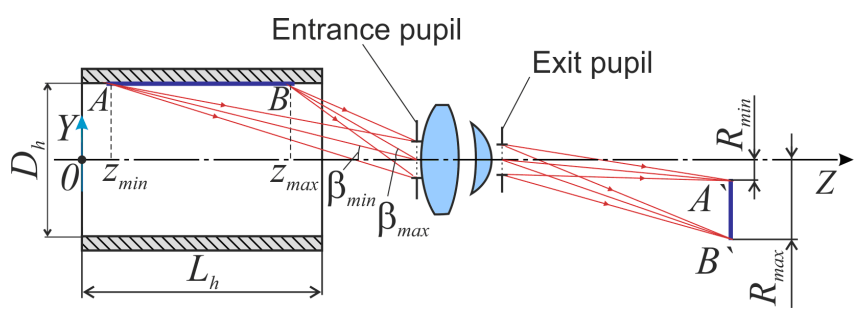

Fig.2. The formation of hole plane image by the specialized inspection lens.

Apparently the $R_{\max } / R_{\min }$ ratio that characterizes the efficiency of photodetector use is approximately equal to $\beta_{\max } / \beta_{\min }$ ratio, where $\beta_{\min }$ and $\beta_{\max }$ are the principal ray angles for points $A$ and $B$ correspondingly. That is why to increase this ratio it is desirable that the HIL is placed closer to the hole or that it has the entrance pupil shifted frontwards.

The hole inspection lens operation can be explained easier, if we consider its operation in the return trace mode. In this case, as well as in the traditional one, there is an ordinary plane object, but its image is strongly incurved (see Fig.3.). When designing with traditional lenses this image curvature is decreased by any means. By contrast, in our example it is necessary to increase the image curvature, so that the curve segment of $A^{\prime} B^{\prime}$ image is placed (situated) as close to the hole surface as possible.

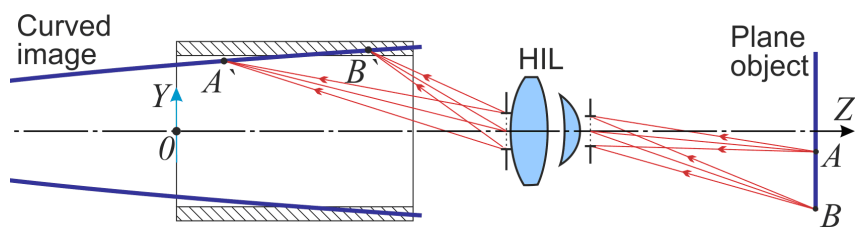

Fig.3. The operation of a hole inspection lens in the return trace mode.
So the task is to design a specialized lens with a high field curvature. By introducing this aberration, the lens corrects the curvature of the conical image of the hole. Evidently, the value of own field curvature of lens should be the higher, the greater the ratio $f^{\prime} / D_{h}$ will be. Thus, the most challenging problem is to design a HIL with a small (5-10 mm) diameter and high $L_{h} / D_{h}$ ratio $\left(L_{h} / D_{h}>1\right)$.

\section{LENS DESIGN FOR INSPECTION OF THE FUEL ASSEMBLY SPACER GRID CELLS}

One of the problems where similar lenses are required is the inspection of the geometrical parameters of the spacer grid cells for nuclear reactor fuel assembly on the base of multiple-ring focusers. In general, solving this problem has been described in papers [6,9]. The main aspects of designing a HIL for solving this problem will be listed below. The method suggested is schematically described in Fig.4.

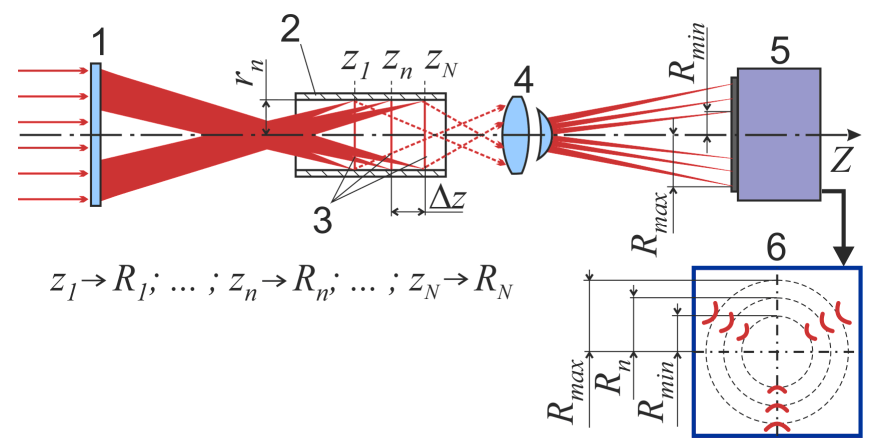

Fig.4. The spacer grid cell inspection method on the base of multiple-ring focusers:

1 - multiple-ring focuser; 2 - cell; 3 - light rings; 4 - HIL; 5 - camera; 6 - image.

This method is based on structural illumination of the cell surface as a set of light circles with the use of a multiplering focuser. A plane image of the cell is formed by the specialized lens with strong curvature.

The most important problem when using this method is the design of a lens to form the plane cell images. Since the diameter of an inspected cell is rather small $\left(D_{h} \approx 9 \mathrm{~mm}\right)$ and its length is rather long ( $\left.L_{h} \approx 15 \mathrm{~mm}\right)$, the application of standard lens does not seem possible.

For this reason, a specialized HIL was designed for spacer grid cells inspection. The calculation of a lens was made using the ZEMAX software. The calculation procedure is based on optimization algorithms of optical system parameters.

As a quality criterion at the initial stage we have used a root-mean-square minimum of geometrical spot from the points throughout the length of the hole.

The following additional requirements were:

- maximal image size $R_{\max }$ (see Fig.4.) is $3.5 \mathrm{~mm}$,

- the photodetector operation efficiency $R_{\max } / R_{\min } \geq 2$,

- the lens F-number $\leq 12$,

- the angle of ray incidence on photodetector should not exceed $10^{\circ}$ (this restriction spreads upon the most existing image sensor types). 
Moreover, there are some restrictions for design parameters of optical elements.

Taking into account simplicity and low cost production considerations, the number of lenses within the HIL was no more than three. Since the lens is supposed to be dealing with laser irradiation, it does not have any requirements on chromatic aberration. Consequently, the glasses optimization was not made under calculations. All the lenses were produced with the use of N-BK7 glasses (the Schott catalogue).

After the first attempts to optimize the system it became clear that image aberration tends to be compressed in the tangential plane (along the $\mathrm{Y}$-coordinate) and extended in sagittal plane (along the $\mathrm{X}$-axis). Then to simplify the optimization process it has been decided to change the optimization criteria from the RMS - Spot Radius to RMS Spot Radius $\mathrm{X}+\mathrm{Y}$, while the weight of the $\mathrm{X}$-component operators was significantly decreased (by five - ten times). Such allowance can be made since the lens is used for registering the bands of structural irradiation, i.e. there are no strict requirements to resolution along the X-axis. After the corrections were made, the lens characteristics were substantially improved. The calculation results are presented in Fig. 5 .

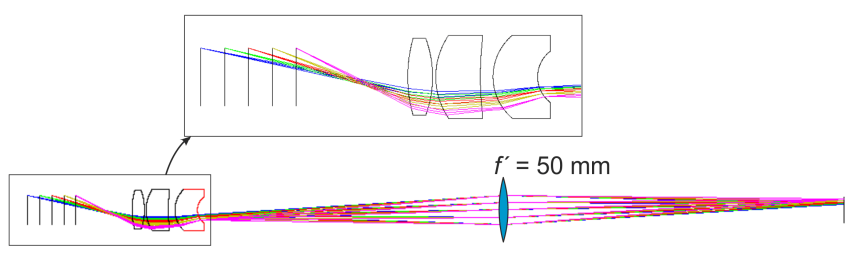

a)

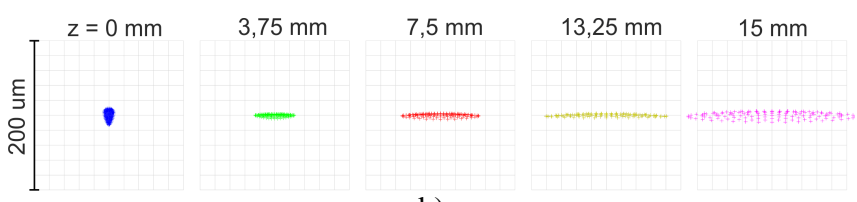

b)

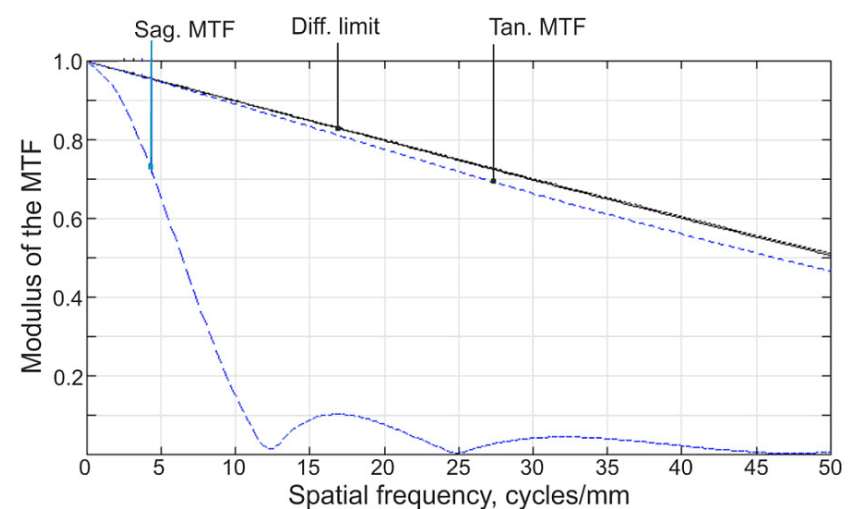

c)

Fig.5. The characteristics of the calculated HIL:

a) the optical scheme, b) the spot diagrams from different points along Z-axis: $0,3.75,7.5,13.25,15 \mathrm{~mm}$; c) the modulation transfer function (for hole center $z=7.5 \mathrm{~mm}$ ).
The optical scheme of the HIL is presented in Fig.5.a). It can be seen that it consists of a double-convex lens and two positive meniscuses. The effective focal length is $f^{\prime}=10.4 \mathrm{~mm}$. Behind the lens group in a distance of approximately $100 \mathrm{~mm}$ there is a standard objective with a focal length of $50 \mathrm{~mm}$ (in ZEMAX it is represented by a thin lens). The standard objective aperture diaphragm is the aperture for the entire optical system, and its object-side image - the entrance pupil - is shifted $8 \mathrm{~mm}$ towards the inspected object and has a diameter of $0.8 \mathrm{~mm}$. In so doing the standard objective projects the hole image (formed near the third lens) on the photodetector of the camera with a magnification of an order $1^{\times}$.

The spot diagrams for various points along the hole axis $\mathrm{Z}$ ( $z=0$ is the point of the hole the most distant from the HIL) are demonstrated in Fig.5.b). Apparently, most of the spot diagrams are extended in sagittal direction (have significant astigmatism). The modulation transfer function (MTF) of an HIL for $z=7.5 \mathrm{~mm}$ in tangential (along the Y-axis) and sagittal (along the $\mathrm{X}$-axis) directions is presented in Fig.5.c). Denoted by a full-line on the upper part of the figure is the MTF for diffraction-limited system (the diffraction limit for this aperture ratio). It is evident that the MTF in tangential direction tends to the diffraction limit. The tangential resolution on the level of 0.5 constitutes 46 cycles per $\mathrm{mm}$.

\section{THE DETAIL ANALYSIS OF THE IMAGING PROPERTIES FOR THE DEVELOPED LENS}

Let us analyze in detail the properties and the operation principle of the obtained optical scheme and try to understand why the correction (compensation) of the hole image curvature occurs (in case of standard lens the hole is supposed to be conical). For this purpose, let us consider the operation of this kind of lens in the return trace mode. The image can now be presented as an even aspherical surface and its parameters can be optimized (the first three aspherical coefficients). The optimization criteria chosen will be the same as when the lens was calculated - Spot $\mathrm{X}+\mathrm{Y}$ with the $\mathrm{X}$-component weight of 0.1 . The result of this optimization is presented in Fig.6. It can be seen that in this case the image is a noticeably extended surface (in the figure it is not fully shown), the shape of which tends to a cylinder one near the inspected hole.

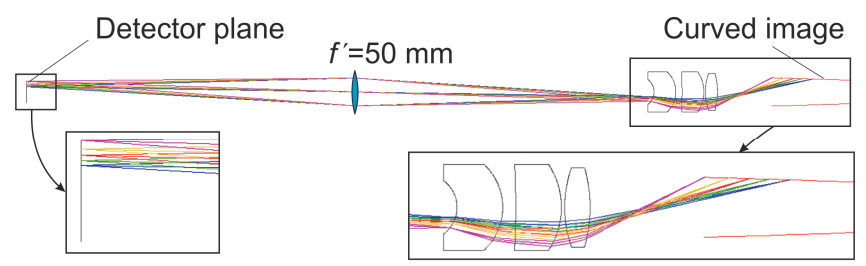

Fig.6. The analysis of HIL operation in the return trace mode.

The designed lens possesses high distortion, as well as significant image curvature and astigmatism. The distortion is the consequence of the shifted frontwards entrance pupil (later we will consider the influence of distortion). The described effect of the lens is the result of great astigmatism and image curvature. 
Now let us define the contribution of which aberration is the principal. The image surface shape presented in Fig.6. is, in fact, the image for tangential beams, because due to optimization the weight of the X-component was minimal. If we optimize the image shape using the criteria of minimal spot radius (or with equal weights of $\mathrm{X}$ and $\mathrm{Y}$ components), then we can define the true image curvature (Fig.7.).

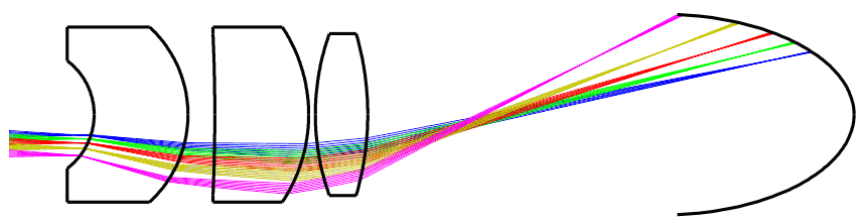

Fig.7. The image curvature for developed HIL.

Thus, comparing Fig.6. and Fig.7., we can see that the own curvature of lens image is not sufficient to observe the hole surface. At the same time, high astigmatism makes a great contribution to the curvature, so the image of tangential beams becomes almost cylindrical.

Let us consider what lenses have influence on astigmatism and image curvature. For descriptive purposes we will present the Seidel coefficients on the lens surfaces as a diagram (see Fig.8., distortion is not shown on the diagram).

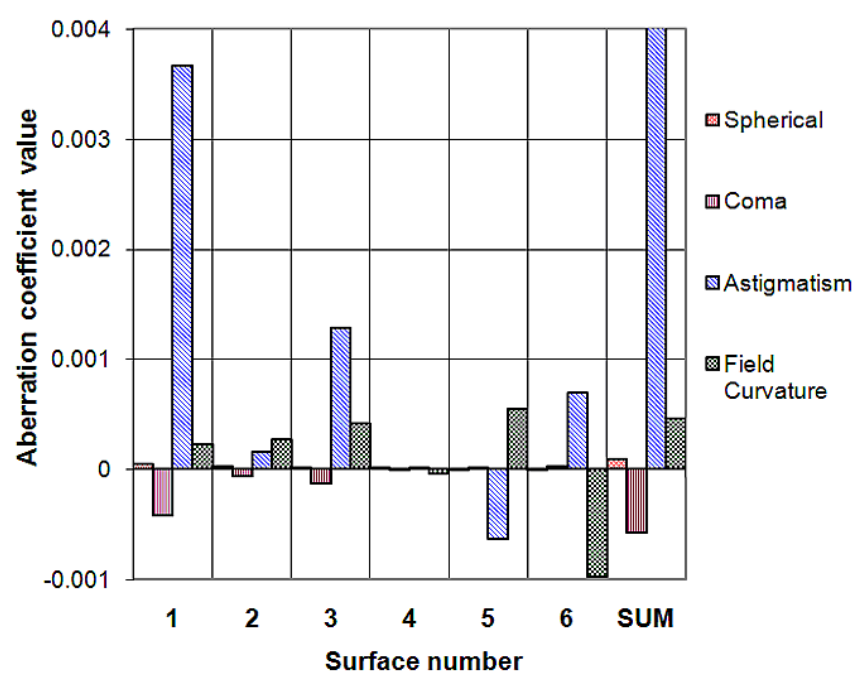

Fig.8. The Seidel diagram of the calculated HIL.

The contribution of each of six surfaces into the four coefficients of the Seidel sum, as well as the overall aberrations of total objective, is demonstrated on the diagram. It is evident from the diagram that the first two lenses form the astigmatism and the image curvature: double-convex lens and a converging meniscus. The last lens - the negative meniscus - decreases insignificantly the coma and increases the astigmatism.

Let us pay some attention to the impact of the distortion phenomenon. As an example we will take nine points, situated with the same intervals between them along the hole, and then we will construct the footprint diagram on the detector (Fig.9.b)). Apparently, the intervals between points on the image become smaller as we get closer to the image center. To estimate the imaging properties we will introduce the term "magnification along the generatrix" (see Fig.9.a)) which for each point of the hole equals:

$$
M_{t}=\frac{d R}{d Z}
$$

The diagram of $M_{t}$ against Z-coordinate is presented in Fig.9.c). It is evident that the magnification along the generatrix, and, correspondingly, the resolution of two extreme points $z=0 \mathrm{~mm}$ and $z=15 \mathrm{~mm}$ differ more than by two times.

It is also worth noting that from resolution increase considerations it is rational to use the astigmatic beams. In fact, if $R_{\max } / R_{\text {min }} \approx 2$, while the hole length is greater than the diameter: $L_{h} / D_{h} \approx 2$, so it can be shown that the resolution in the meridional direction should be much higher than in the sagittal one. For example, the mean magnification value along the hole:

$$
M_{t}=\frac{R_{\max }-R_{\min }}{L_{h}}=\frac{R_{\min }}{L_{h}}
$$

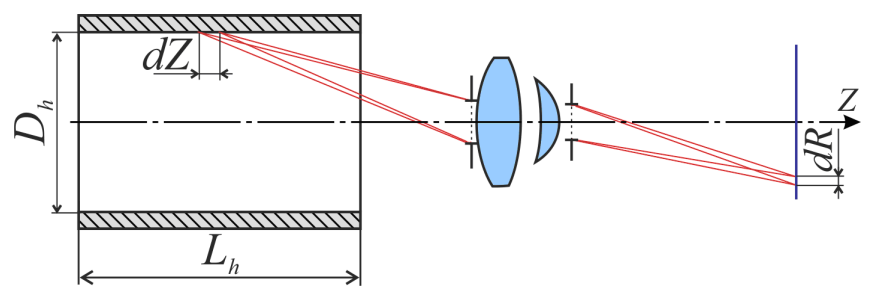

a)

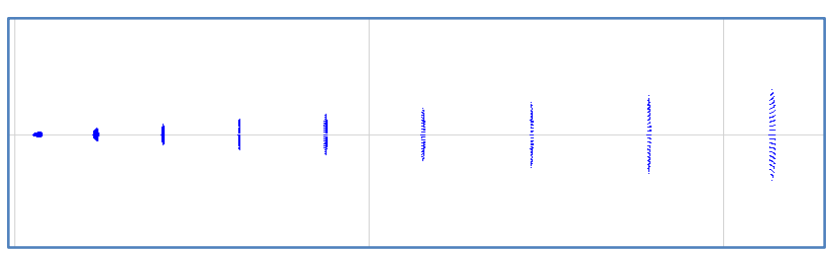

b)

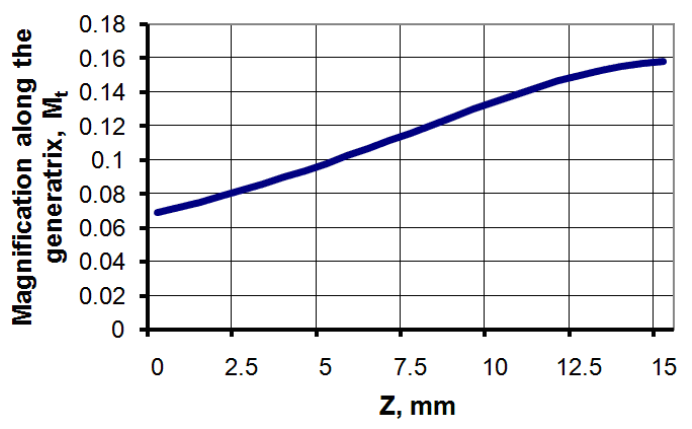

c)

Fig.9. The HIL distortion.

a) to the illustration of term «magnification along the generatrix», b) the footprint diagram of the points situated with same intervals between them along the hole, c) the diagram of $M_{t}$ along Z-coordinate. 
At that, for the hole center a circular curve with a length of $\pi D_{h}$ is imaged into a circular curve with a length of $2 \pi\left(R_{\max }+R_{\min }\right) / 2$, and the magnification in sagittal direction is equal to:

$$
M_{s}=\frac{\frac{2 \pi\left[R_{\max }+R_{\min }\right]}{2}}{\pi D_{h}}=6 \frac{R_{\min }}{L_{h}}=6 M_{t}
$$

Thus, it is evident that when calculating such lenses it is essential to consider the significant distortions of hole images and to use various weight coefficients for tangential and sagittal quality criteria (especially for ratio $L_{h} / D_{h}>1$ ).

The image of a spacer grid fragment is shown in Fig. 10 . An image of a cell of this spacer in white light (a) and with structural illumination (b), obtained with the use of the designed HIL is presented in Fig.11. Three bumps close to the image center are the so called protrusions, holding the fuel elements together. It can be seen that the image of the cell throughout the depth $(\sim 20 \mathrm{~mm})$ is clearly displayed. And at the same time the cell image looks as everted because of the HIL large distortion.

The lens calculation results also demonstrate that it can be used to inspect holes within a rather great range of diameters from $8 \mathrm{~mm}$ to $20 \mathrm{~mm}$. Moreover, the larger the hole diameter, the higher the image quality. The readjustment of the HIL for other hole diameters consists of changing the distances between the hole and the HIL and between the photodetector and the HIL.

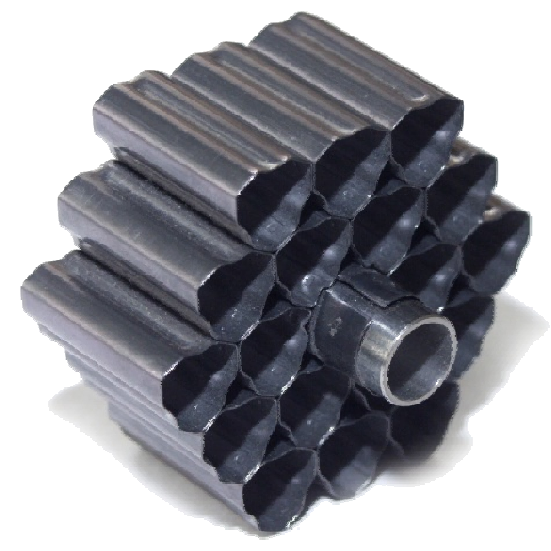

Fig.10. Spacer grid fragment.

One of the disadvantages of the designed lens is its small aperture, that is slightly reduced (working F-number 12) due to rather high aberration level. It might be explained by the simplicity of the HIL construction: only three lenses are used and no aspherical surfaces. Under further development of HIL with greater number of lenses this disadvantage might be eliminated.

It also should be noted that the development of lenses with several (i.e. two or more) intermediate images will allow reaching better image quality for holes with small diameter and/or great $L_{h} / D_{h}$ ratio. In this case, it is possible to achieve greater cumulative field curvature without the substantial beam astigmatism.

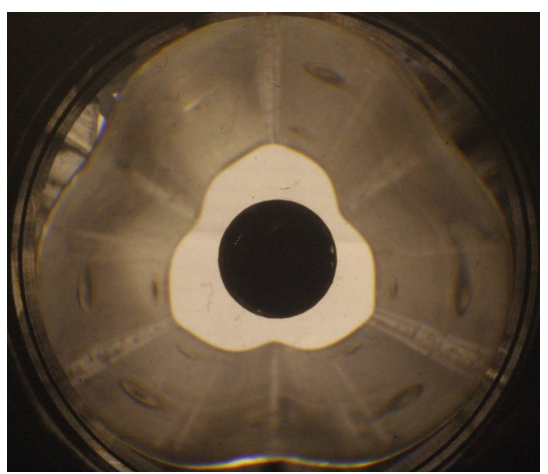

a)

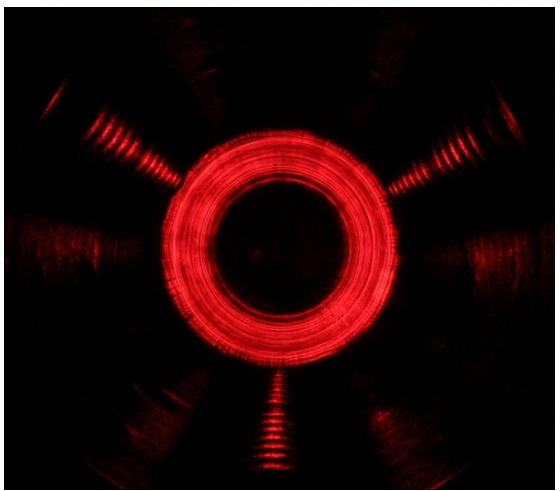

b)

Fig.11. The images of spacer grid cell, obtained with the use of the designed HIL:

a) in white light, b) with structural illumination.

\section{CONCLUSION}

The method for calculating the hole inspection lens with high field curvature (as a triple lens) is presented. The hole inspection lens forming of plane images of the 3D inner surfaces of the spacer grid cells was calculated and the corresponding lens type was produced. The analysis of the imaging properties of calculated HIL was performed. Unlike standard lenses, this kind of lens has a greater field curvature in the object space, which is achieved at the expense of greater astigmatism. Due to increased aberrations the lens forms a plane image of the holes' inner surfaces within a broad range of their diameters $D_{h}(8-20 \mathrm{~mm})$ and lengths $L_{h} \leq(1 \div 2) \times D_{h}$ with spatial resolution in tangential and sagittal directions on 45 and 8 cycles $/ \mathrm{mm}$ correspondingly.

Using this lens makes it possible to simplify the spacer grid inspection scheme significantly, to increase the measurement accuracy while measuring the geometrical parameters of cells, and, lastly, to substantially broaden the nomenclature of the measured spacer grids as compared to the inspection system designed earlier, where standard lenses had been applied $[11,12]$.

\section{ACKNOWLEDGMENTS}

This material is based upon work supported by the Ministry of Education and Science of the Russian Federation under Grant № 14.604.21.0086. The author also wishes to thank Prof. Yuri Chugui for his assistance during this paper writing. 


\section{REFERENCES}

[1] Wakayama, T., Machi, K., Yoshizawa, T. (2012). Small size probe for inner profile measurement of pipes using optical fiber ring beam device. In Optical Metrology and Inspection for Industrial Applications II, Proc. SPIE 8563.

[2] Bao Hua Zhuang, Wenwei Zhang, Dong Yin Cui. (1997). Noncontact laser sensor for pipe inner-wall inspection using circular optical cutting method. In Three-Dimensional Imaging and Laser-Based Systems for Metrology and Inspection II, Proc. SPIE 2909, 223.

[3] Shi Yongqiang, Sun Changku, Ma Yukun, Duan Hongxu, Wang Peng. (2012). High-precision automatic online measurement system of engine block top surface holes. Optical Engineering, 51 (5), 3604.

[4] Chugui, Yu., Finogenov, L., Kiryanov, V., Nikitin, V., Sametov, A., Zavyalov, P. (2004). Inspection of holes parameters using a ring diffractive focuser. In Photonics in Measurement, 23-24 June 2004. Düsseldorf: VDI Verlag, 433-443.

[5] Chugui, Yu.V., Lemeshko, Yu.A., Zav'yalov, P.S. (2009). Application of diffractive optical elements for inspection of complicated through holes. In Fifth International Symposium on Instrumentation Science and Technology, Proc. SPIE 7133.

[6] Finogenov, L.V., Lemeshko, Yu.A., Zav'yalov, P.S., Chugui, Yu.V. (2007). 3D laser inspection of fuel assembly grid spacers for nuclear reactors based on diffraction optical elements. Measurement Science and Technology, 18 (6), 1779-1785.
[7] Vertoprakhov, V., Tian Poh Yew. (2011). Hole Inspection Method and Apparatus. Patent US20110128368 A1.

[8] Opto Engineering. Hole inspection optics for $360^{\circ}$ inside view in perfect focus. http://www.optoengineering.com/products/pchi-hole-inspection-optics.

[9] Lemeshko, Yu.A., Finogenov, L.V., Zav’yalov, P.S. (2008). Using the diffractive optics for 3D inspection of nuclear reactor fuel assembly grid spacers. Measurement Science Review, 8 (3), 74-77.

[10] Zemax LLC. Zemax Optical and Illumination Design Software. http://www.zemax.com/products/opticstudio.

[11] Bityutskii, O.I., Vertoprakhov, V.V., Gushchina, A.A. et al. (2003). Three-dimensional noncontact inspection of geometric parameters of grid spacers in nuclear reactors. Optoelectronics, Instrumentation and Data Processing, 39 (5), 4.

[12] Bityutsky, O.I., Chapaev, I.G., Chernyshov, V.M. et al. (2002). Laser measuring machine for 3D noncontact inspection of geometric parameters of grid spacers for nuclear reactors VVER-1000. In Seventh International Symposium on Laser Metrology Applied to Science, Industry, and Everyday Life, Proc. SPIE 4900, 202.

Received October 7, 2014. Accepted February 4, 2015. 